\title{
Jochen Vogt: \\ Was aus dem Mädchen geworden ist. Kleine Archäologie eines Gelegenheitstextes von Anna Seghers
}

Die Stadt Mainz liegt auf der linken Rheinseite, ihr gegenüber mündet der Main in den Strom. Mainz ist ein wichtiger Eisenbahnknotenpunkt und liegt an der Hauptstrecke, die, dem Verlauf des Rheintals folgend, von Holland in die Schweiz führt. In Mainz überqueren die Haupteisenbahnlinien nach Frankfurt am Main und nach Mannheim den Rhein. Die Stadt besitzt einen Binnenhafen und ist einer der größten Güterumschlagplätze am Oberrhein. Die in der Stadt ansässigen Industriebetriebe stellen Maschinen, Eisenbahnwaggons und Schiffe her. Mainz ist das Zentrum des Rheinweinhandels und der deutschen Schaumweinindustrie.

(The Bomber's Baedeker: Guide to the Economic Importance of German Towns and Cities, 2nd Edition, Part 1, 1944)

Sie erzählt mit Pausen wie einer, der auf die berufenen Hörer im Stillen wartet und, um Zeit zu gewinnen, manchmal innehält. 'Je später auf den Abend, desto schöner die Gäste'. Diese Spannung durchzieht das Buch. Es ist weit entfernt von der Promptheit der Reportage, die nicht viel nachfragt, an wen sie sich eigentlich wendet. Es ist ebenso weit entfernt vom Roman, der im Grunde nur an den Leser denkt. Die Stimme der Erzählerin hat nicht abgedankt. Viele Geschichten sind in das Buch eingesprengt, welche darin auf den Hörer warten.

(Walter Benjamin: Eine Chronik der deutschen Arbeitslosen. Über Anna Seghers Roman Die Rettung, 1938)

Ich erinnere mich an einen heißen Sommertag in Stockholm, als wir, Anna Seghers, Franz Dahlem und ich, zu dritt einen Hügel zu einem Restaurant emporstiegen. Dahlem war uns einige Schritte voraus, und sie sagte auf einmal, dicht neben mir, scheinbar ohne jeden Zusammenhang "Mein geliebtes jüdisches Volk..." Ich sagte nichts. Ich begriff alles - daß sie an ihre Mutter dachte, die schutzlose alte Frau, die im Konzentrationslager Piaski in Polen ihr Ende gefunden hatte; auch daß wir nicht mehr in der Fremde waren und dennoch nicht in der Heimat. Ich wußte, daß Anna Seghers tief in ihrem Innern unter Bergen von Schweigen Worte und Schreie barg, die niemals laut wurden. 
(Stephan Hermlin: Nachdenken über Anna Seghers, 1990/92)

Mit den nachfolgenden Beobachtungen und Überlegungen möchte ich die Aufmerksamkeit auf einen kleinen Text lenken, den die etablierte Seghers-Forschung bislang ignoriert oder allenfalls beiläufig erwähnt hat. Im Findbuch des Anna Seghers-Archivs zu Berlin ist er unter der irreführenden Genrebezeichung "Aufsatz" aufgeführt, in Sigrid Bocks maßgeblicher SeghersEdition Über Kunstwerk und Wirklichkeit findet er sich im Ergänzungsband unter der Kategorie "Aufsätze, Reden, Notate u.a."; beide Male wird ein Mainzer Nachdruck von 1973 fälschlich als Erstveröffentlichung angenommen ${ }^{1}$. In Darstellungen zu Leben und Werk der Autorin wird er bisweilen zur atmosphärischen Charakterisierung der Jugendzeit und als Beleg für Netty Reilings frühe künstlerische Sensibilität zitiert. ${ }^{2}$ Das ist nicht falsch, aber bei weitem nicht die ganze Wahrheit. Ich sehe dagegen in der autobiographischen Skizze Zwei Denkmäler ${ }^{3}$ von 1965 ein Prosastück von dichterischem Rang, bei aller Kürze von hoher thematischer und struktureller Komplexität, gewissermaßen einen "Gelegenheitstext" - mit allen goetheschen Anklängen ${ }^{4}$ des Begriffs; einen Schlüsseltext, der zentrale Seghersche Motive anspricht (oder durch Verschweigen deutlich macht) und deshalb auch als Einladung zur Entdeckung dieser Autorin und zur Lektüre ihres Erzählwerks genutzt werden kann. ${ }^{5}$ Diese meine Einschätzung möchte ich im folgenden (with a little help from my friends) untermauern, indem ich den Text zunächst einem kleinen narratologischen Exerzitium (I) unterziehe, um seine Erzählstrategie zu

\footnotetext{
${ }^{1}$ Im Findbuch des Archivs ist eine maschinenschriftliche Kopie mit handschriftlichen Korrekturen von fremder Hand nachgewiesen. Vgl. Anna Seghers: Über Kritik und Wirklichkeit. Bd. IV: Ergänzungsband. Bearbeitet und eingeleitet von Sigrid Bock, Berlin (Akademie-Verlag) 1979, S. 102f. Dort S. 222 der Quellennachweis: Anna Seghers aus Mainz. Kleine Mainzer Bücherei. Mainz 1973, S. 75f. - Diese Datierung wird u.a. noch beim Abdruck einer kurzen Textstelle in der jüngsten Bildbiographie übernommen: Anna Seghers. Eine Biographie in Bildern, hrsg. von Frank Wagner, Ursula Emmerich, Ruth Radvanyi, Weimar/Berlin 1994, S. 24.

${ }^{2}$ Vgl. z.B. Kurt Batt: Anna Seghers. Versuch über Entwicklung und Werke, Leipzig 1980, mit korrekter Datierung auf 1965! - In den neuesten Einführungen ins Gesamtwerk - Andreas Schrade: Anna Seghers, Stuttgart/Weimar 1993, und Christiane Zehl-Romero: Anna Seghers, Reinbek 1994 - ist der Text, soweit ich sehe, nicht erwähnt.

${ }^{3}$ Derzeit in folgenden Textsammlungen bequem greifbar: Deutsche Literatur der sechziger Jahre. Ein Lesebuch, hrsg. v. Klaus Wagenbach, Neuausgabe Berlin 1996; Vom Nullpunkt zur Wende. Deutschsprachige Literatur 1945-1990, hrsg. v. Hannes Krauss, Essen 1994; Anna Seghers: Die Heimkehr des verlorenen Volkes. Ein Lesebuch, hrsg. von Sonja Hilzinger, München 1996.

${ }^{4}$ Vgl. besonders Goethes Selbstkommentar zu "Harzreise im Winter" (Hamburger Ausgabe, Bd. 1, München 1978, S. 372ff.) und den Metakommentar von Albrecht Schöne (Götterzeichen, Liebeszauber, Satanskult. Neue Einblicke in alte Goethetexte, München 1982, S. 15f.), der - mutatis mutandi - auch meine Sicht auf Seghers' alte Texte bestärkt, wenn nicht gar angeregt hat.

${ }^{5} \mathrm{Zu}$ literaturdidaktischen Möglichkeiten bei der Behandlung dieses Textes im Unterricht vgl. meinen Aufsatz "Geschichte(n) eines Adjektivs. Was man mit einem kleinen Text von Anna Seghers machen kann", in: Der Deutschunterricht, H.4/1997: Kinder und der Holocaust.
} 
verdeutlichen, sodann einige lebens- und werkgeschichtliche Kontexte (II-V) zu klären suche, die für die Textkonstitution wichtig sind, und aus diesen Beobachtungen schließlich eine These zur Interpretation (VI) und einige weiterführende Überlegungen (VII) entwickle.

\section{I. "Wer erzählt hier eigentlich?"}

In diesem Fall, ausnahmsweise einmal, die Autorin selbst. Der Text gibt keinerlei Indizien, die die Identität der Ich-Figur im Text, der Ich-Erzählerin und der Autorin Anna Seghers in Frage stellen könnten. ${ }^{6}$ Wir haben eine faktuale Erzählung ${ }^{7}$ vor uns, genauer: eine autobiographische Skizze, in der die Perioden und Zäsuren deutscher Geschichte im vergangenen Jahrhundert klar hervortreten: Kaiserreich, Erster Weltkrieg, Weimarer Zeit und Exil, Zweiter Weltkrieg, Nachkriegsdeutschland. Das "Ich" der Erzählung, die erste Person Singular, umfaßt in diesem Text neben dem erinnernden nicht nur ein erinnertes Ich, sondern gleich mehrere, - die jeweils verschiedene Lebensalter der Autorin/Protagonistin von der Schulzeit um 1910 bis zur Schreibgegenwart Mitte der sechziger Jahre markieren. Damit ist die erste von drei narrativen Strukturen erfaßt, deren Zusammenspiel die Erzählstrategie dieses Textes - und sein dichterisches Wirkungspotential - ausmacht. Wir könnten sie Zeitschichtung nennen (und beiläufig daran erinnern, daß Christa Wolf als erste von "Zeitschichten" $\operatorname{sprach}^{8}$, um das Erzählprinzip der Seghers zu charakterisieren). Der Erzählprozeß entwickelt sich nun, indem er vom Zeit-Punkt der Niederschrift aus auf verschiedene Ebenen der Vergangenheit (grammatisch: Imperfekt, Perfekt) zurückgreift, um zwischendurch immer wieder in die Schreibgegenwart (grammatisch: Präsens) zurückzukehren. Streng narratologisch handelt es sich um ein Spiel von Anachronien innerhalb der temporalen Ordnung, also von "verschiedenen Formen der Dissonanz zwischen der Ordnung der Geschichte und der der Erzählung". ${ }^{9}$ Wie dieses Spiel in Zwei Denkmäler funktioniert, habe ich an anderer Stelle in Form eines detaillierten Schemas gezeigt, das Satz für Satz, manchmal Wort für Wort, diese Zeitebenen definiert und das Spiel der Erinnerung (denn darum handelt es sich bei einer Ich-Erzählung offensichtlich) als "Zeitreise" anschaulich macht. ${ }^{10}$ Hier will ich nur zusammenfassen, daß Zwei Denkmäler eine relativ regelmäßige Pendelbewegung zwischen der

\footnotetext{
${ }^{6}$ Vgl. Philippe Lejeune: Der autobiographische Pakt, Frankfurt/M. 1994, S. 14f.

${ }^{7}$ Vgl. Gérard Genette: Fiktion und Diktion, München 1992, S. $65 \mathrm{ff}$.

${ }^{8}$ Christa Wolf: Zeitschichten, in: Anna Seghers. Ausgewählte Erzählungen, hrsg. u. mit einem Nachwort von Ch.W., Darmstadt/Neuwied 1983, S. 363ff.

${ }^{9}$ Gérard Genette: Die Erzählung. Hrsg. u. mit einem Nachwort von Jochen Vogt, München 1994, S. 23.
} 
Schreibgegenwart und mehreren (fünf bis sechs) Stufen der erinnerten Vergangenheit inszeniert, ein regelrechtes "Zickzack der Erinnerung". (Das ist an sich kein ungewöhnliches Verfahren; bemerkenswert bleibt jedoch, auf welch knappem Erzählraum es hier durchgeführt wird; Marcel Prousts Romanwerk, an dem Gérard Genette diese "Zickzackbewegung"11 exemplarisch analysiert hat, ist etwa viertausendmal so lang wie Zwei Denkmäler.)

Mit der Zeitschichtung verbunden ist in unserem Text eine leicht 'dezentrierte' Erzählsituation: Die Erzählerin erscheint als Figur in ihrer Geschichte, aber ist sie die Hauptfigur? Gewiß, ihr ganzes Leben ist in den Erinnerungsrückblick eingespiegelt; aber andererseits wird es doch auch (vor allem? nur?) erzählt, um der Episode vom Tod der Frau Eppstein und den sich anschließenden Fragen einen Rahmen zu schaffen. Ich sehe in dieser Episode den thematischen Erzählkern - und im Hinblick darauf die autobiographische Erzählerin auch in der Rolle der Chronistin. ${ }^{12}$ Narratologisch wird diese Variante der Ich-Erzählung üblicherweise "I as a witness" oder "je témoin" genannt ${ }^{13}$ - das Ich als Zeuge oder Zeugin, was man in diesem Fall nicht nur funktional als "Augenzeugin", sondern auch inhaltlich als "Zeitzeugin" verstehen darf.

Als zweite signifikante Erzählstruktur ist das abstrakte "Konzept der Geschichte" (im Sinne von Greimas oder Stierle ${ }^{14}$ ) hervorzuheben: in diesem Fall die durchgängige Opposition von Groß und Klein, Oben und Unten, Macht und Ohnmacht, die ja schon im Titel angelegt ist und sich vor allem auf der Ebene der Dingsymbole (Dom vs. Gedenkstein) entfaltet. Sie verbindet allerdings auch den autobiographischen Rahmen und die zentrale Binnenepisode miteinander und bringt schließlich auch eine unverwechselbare Signatur von Seghers' Geschichtsauffassung zum Ausdruck, die mit der Formel Die Kraft der Schwachen bezeichnet werden könnte - das ist bezeichnenderweise ein Titel aus dem gleichen Jahr 1965, in dem auch Zwei Denkmäler erschien! Diese paradigmatische Opposition lädt Leser und Leserinnen erfahrungsgemäß zu eigenen, durchaus unterschiedlichen Lesarten ein und leitet so zur dritten, wirkungsästhetisch besonders wichtigen Erzählstruktur über. Es geht um die beiden Unbestimmtheits- oder Leerstellen (im

\footnotetext{
${ }^{10}$ Jochen Vogt: Aspekte erzählender Prosa. Eine Einführung in Erzähltechnik und Romantheorie. 7. Aufl. Opladen 1990, S. 130ff.

${ }^{11}$ Vgl. Genette: Die Erzählung, S. 30.

${ }^{12}$ Zum chronikalischen Zug bei Seghers: Walter Benjamin: Eine Chronik der deutschen Arbeitslosen. Zu Anna Seghers Roman "Die Rettung" (1938), in: W.B.: Gesammelte Schriften, Bd. III, Frankfurt/M. 1972, S. 530ff.

${ }^{13}$ Vgl. Vogt: Aspekte erzählender Prosa, S. 75.

${ }^{14}$ Karlheinz Stierle: Geschehen, Geschichte, Text der Geschichte, in: K.S.: Text als Handlung, München 1975, S. 49ff.
} 
Sinne Ingardens, Isers oder Ecos ${ }^{15}$ ) um den eigentümlich offenen Doppelschluß unseres Textes, der die Fortschreibung der erzählten, oder genauer eben: der nicht erzählten Geschichte in der Lektüre anregt, erfordert, provoziert. Was geschah mit dem zweiten Denkmal? Und was ist aus dem Mädchen geworden? Mit diesen Fragen - und dem unausgesprochenen Appell, selbst die fehlenden Antworten zu finden - läßt die Autorin ihre Leser und Leserinnen am Schluß zurück, so daß wir die Frage Wer erzählt hier eigentlich?, die aus Uwe Johnsons Jahrestagen entliehen ist, ähnlich wie dort Gesine Cresspahl beantworten könnten: Wir beide. Das weißt du doch, Seghers. ${ }^{16}$

\section{Was ist wirklich geschehen?}

Eine Frage, die in der Literaturwissenschaft heutzutage eher verpönt ist, angesichts dieses autobiographischen, vielfältig in der Zeitgeschichte verwurzelten Textes aber wohl doch gestellt werden darf. Ein kurzer Besuch im Mainzer Stadtarchiv liefert uns die Antwort - in Form einer Verlautbarung des Polizeiamts vom 10. März 1918, Betreffend: Fliegerangriff auf Mainz am 9. März 1918:

"Gestern Nachmittag gegen $1 \frac{1}{2}$ Uhr wurden in Mainz und Umgebung von einem feindlichen, vermutlich englischen Fliegergeschwader eine größere Anzahl Bomben abgeworfen, die neben bedeutendem Sachschaden, auch vielfach leichte und schwere Verletzungen von Personen sowie leider auch den Tod von 4 Militärpersonen und 7 Zivilpersonen herbeigeführt haben. Unter den getöten Zivilpersonen befinden sich 2 männliche und 4 weibliche Erwachsene sowie ein Knabe." Namentlich aufgeführt werden diese dann als Karl Codini, Wirt; Heinrich Wolf, Steuermann; Adam Hofmann [14 Jahre], Sohn [eines] Tagelöhners; Meta Cahn, Ehefrau; Maria Willmuth, Ehefrau; Maria Mappes und Katharina Winsiffer, beide Dienstmädchen." - Auf dieser Verlautbarung basieren schließlich auch die fast gleichlautenden Meldungen in allen örtlichen Tageszeitungen. ${ }^{17}$

\footnotetext{
${ }^{15}$ Vgl. Jochen Vogt: Grundlagen narrativer Texte, in: Grundzüge der Literaturwissenschaft, hrsg. von Heinz Ludwig Arnold und Heinrich Detering, München 1996, S. 306f.

${ }^{16}$ Vgl. Uwe Johnson: Jahrestage. Aus dem Leben von Gesine Cresspahl, Bd.1, Frankfurt/M. 1970, S. 256.

17 Vgl. etwa "Fliegerangriff auf Mainz", in: Mainzer Anzeiger, 11. März 1918, Rubrik "Lokales", und "FliegerAngriff auf Mainz", in: "Volkszeitung", 11. März 1918, Lokalbeilage. - Unter dem gleichen Datum wird, jeweils auf der ersten Seite, nicht nur vom "Rücktritt Trotzkis" (als "Volksbeauftragter für auswärtige Angelegenheiten" der Sowjetunion) berichtet (Mainzer Anzeiger, 11. 3. 1918), sondern etwa auch über einen (englischen) "Flieger-Angriff auf Koblenz" sowie (deutsche) "Luftkrieg-Erfolge gegen England", "Luftangriffe auf Paris" und "Bomben auf Neapel" (Volkszeitung, 11. 3. 1918). In Mainzer Kinos wird das Lustspiel "Hedda im Bade" eingerahmt von dokumentarischen Bildern über den "Höllenkampf an der Aisne: Hochinteressante militärische Aufnahmen, darunter u.a. ein Fliegerkampf" (Mainzer Anzeiger, 11. 3. 1918).
} 
Die "zwei jungen Dienstmädchen" waren im Hause des Verlegers Eduard Goldschmidt in der Schulstraße beschäftigt und haben ein bescheidenes Epitaph in den Memoiren seines Enkels Carl Zuckmayer gefunden. ${ }^{18}$ Aber vor allem sollte uns hier Frau Cahn interessieren.

Meta Cahn, geb. Altmann, aus Karlsruhe war verheiratet mit Jacob Cahn, der von seinem Vater Herz Cahn eine Druckerei und Papiergroßhandlung am Bonifaziusplatz geerbt hatte. Die Familie wohnte wenige Minuten entfernt in der Schulstraße Nr. 54, in einer gründerzeitlichgutbürgerlichen Wohngegend. Die Cahns waren ähnlich gutsituiert und gesellschaftlich anerkannt wie die Familie Reiling, die in der parallel verlaufenden Kaiserstraße Nr. 34 geradezu "herrschaftlich" wohnte ${ }^{19}$ und mit den Cahns gut bekannt war. Auch die öffentliche Reaktion nach Meta Cahns Tod zeugt von allgemeiner Wertschätzung, wenn wir dem Neuesten Anzeiger vom 12. März glauben dürfen: "Die erste Beisetzung der Opfer, der Frau Meta Cahn, erfolgte gestern nachmittag. Herr Oberbürgermeister Dr. Göttelmann gab seiner Teilnahme in ergreifenden Worten Ausdruck. Die ganze Stadt vereinigte sich in der Trauer, da ja jeder getroffen werden könne. Rabbiner Dr. Bondi widmete dem Kind seiner Gemeinde einen tränenerfüllten Nachruf."

Eine typische Passage aus der Ansprache des Oberbürgermeisters macht den historischideologischen Deutungshorizont anschaulich, in dem dieses Totengedenken gestiftet wird: "Und auch die Toten, denen wir heute die letzte Ehre erweisen, sind gefallen fürs Vaterland. Ihrer wollen wir mit gleicher Liebe gedenken, und ihre Gräber sollen ebenso in Ehren gehalten und geschmückt werden, wie die unserer gefallenen Krieger. [...] Es ist nicht unsere Schuld, daß es so kam, denn wir haben den Krieg nicht gewollt und hätten ihn längst beendet, wenn man unsere zum Frieden ausgestreckte Hand nicht mit Hohn zurückgewiesen hätte. Wir haben auch nicht damit begonnen, den Krieg auf die friedlichen Einwohner der feindlichen Länder auszudehnen, nachdem aber unsere Feinde damit angefangen haben, müssen wir aus Notwehr Gleiches mit Gleichem vergelten. So sind wir denn alle Kämpfer geworden, sie draußen, wir hier im Innern, müssen mit ihnen dulden und aushalten und dürfen nicht verzagen. [...] Einmal aber wird die Sonne des Friedens über den Völkern wieder aufgehen. Ach, daß sie bald erschiene! Dann, so hoffen wir, wird ein milderes, glücklicheres Zeitalter kommen, das uns die heutige schwere Not

\footnotetext{
${ }^{18}$ Vgl. Carl Zuckmayer: Als wär's ein Stück von mir. Horen der Freundschaft, Frankfurt/M. 1996, S. $194 f$.

${ }^{19}$ Vgl. Friedrich Schütz: Die Familie Seghers-Reiling und das jüdische Mainz, in: Argonautenschiff 2 (1993), S. 151ff., besonders S. 157ff.
} 
der Zeit wird vergessen lassen und uns durch das Glück unseres Volkes die schweren Opfer lohnen wird, die wir heute bringen müssen." ${ }^{20}$

Auch das allergnädigste Beileidstelegramm des Großherzogspaares von Hessen-Darmstadt wird in der Presse ausführlich gewürdigt. Das Grab von Meta Cahn ist, ebenso wie dasjenige von Anna Seghers' Vater Isidor Reiling, noch heute auf dem Jüdischen Friedhof in Mainz zu finden.

Der Witwer Jacob Cahn konnte 1939, nach den üblichen massiven Repressalien durch die Nazis, nach Israel gelangen, wo er im Alter von 92 Jahren 1975 verstorben ist. ${ }^{21}$ Kurz zuvor hatte er einer Mainzer Chronistin noch seine Erinnerung an den Schicksalstag von 1918 mitgeteilt: "Ich war mit meiner Frau an diesem Samstag auf dem Weg von meinen auf dem Bonifaziusplatz wohnenden Eltern zu unserer Wohnung in der Schulstraße, als die erste Bombe fiel. Wir hielten es erst für Fliegeralarm, aber es war schon ernst (angeblich sei bei der Alarmstelle Mittagspause gewesen) und meine Frau rief: 'Ich lauf zu den Kindern', als auch schon die zweite Bombe niederging, an der Ecke zum Bonifaziusplatz, und sie tödlich traf. Ich war nur etwa 10 Meter hinter ihr, konnte ihr nicht schnell genug folgen, und blieb unverletzt. Wir hatten zwei Kinder, ein Junge von noch nicht vier und ein Mädel von eineinhalb Jahren. [...] An dem Stein, den man in das beschädigte Pflaster der Ungücksstelle eingesetzt hat, bin ich jahrelang - bis wir [...] in die Walpoldenstraße umzogen - auf meinen täglichen Weg in mein Geschäft auf dem Bonifaziusplatz vorbeigegangen, es war eine ovale Platte aus kleinen hellen und dunklen Steinen zusammengesetzt, aber ein Name war nicht darauf angebracht." 22

Ein junger Forscher namens Christoph Bathelt, Schüler am Rabanus-Maurus-Gymnasium, zwischen Kaiser- und Schulstraße gelegen, hat noch vor kurzem einen Briefwechsel mit Meta und Jacob Cahns Tochter, Frau Margarete Oppenheimer geführt. In einem Brief aus Buenos Aires rekapituliert sie am 1. Februar 1993 nochmals die Schilderung ihres Vaters und zieht eine Linie bis in die Gegenwart: "Ich war im Juni 1992 zu einer Begegnungswoche in Mainz und sprach bei dieser Gelegenheit vor einer Klasse des früheren Realgymnasiums in der Schulstrasse, jetzt Adam-Karrillonstr. Wir wohnten früher gegenüber von dieser Schule, Schulstr. 54. Bei Wahlen wurde die Schule zum Wahllokal und wir schauten zum Fenster hinaus, um zu sehen, ob

\footnotetext{
${ }^{20}$ Mainzer Journal, 15. 3. 1918.

${ }^{21}$ Vgl. Barbara Glauert: Jacob Cahn zum Gruß, in: Das neue Mainz, H.11/1973, S. 2f.; weiterhin: Jacob Cahn: Jugend in der Betzelsgasse. Erinnerungen an das Mainz um die Jahrhundertwende, in: Das neue Mainz, H.11/1962, S. 7f.

${ }^{22}$ Zitiert nach: Barbara Glauert: Über ein vergessenes Denkmal in Mainz. Erinnerungen an Meta Cahn, in: Das Neue Mainz, H.3/1973, S. 2f.
} 
Bekannte kamen, um zu wählen. Ich erinnere mich an die Plakate, die für Ebert und Hindenburg warben... und später für Hitler." 23

Damit sind einige Fragen historischer Natur beantwortet, die Zwei Denkmäler aufwirft, nicht zuletzt die im letzten Satz angesprochene: was aus dem Mädchen geworden ist? Zugleich aber stellen sich neue - und spezifisch literarische - Fragen an den Text: Trug der Gedenkstein einen Namen oder nicht? Warum heißt Frau Cahn dort Eppstein? Warum kann die Autorin sich an den Namen der Straße nicht mehr erinnern und den Stein "bei [ihren] Besuchen" in der Nachkriegszeit nicht mehr finden? Und warum verschweigt sie uns, was aus dem Mädchen wirklich geworden ist?

Tatsächlich hatte man wohl zunächst eine Steinplatte mit einem Kreuz verlegt, um die mindestens vier Opfer in der Schulstraße zu ehren, dann aber - offenbar aus Rücksicht auf die jüdische Mitbürgerin - durch das schwarz-weiße Mosaik ersetzt, das man in der inzwischen nach dem Regionaldichter Adam Karrillon ${ }^{24}$ benannten Straße vor dem Haus Nr. 23 leicht finden kann (und wohl auch immer finden konnte). ${ }^{25}$ Die Umbenennung der Figur hat Seghers selbst 1969 in einem Brief an Jacob Cahn in Jerusalem erklärt: "Ich habe absichtlich einen beliebigen fremden Namen gewählt, weil ich vielleicht die Schilderung, die Sie kennen, als Anfang einer längeren Erzählung benutzt hätte, in der gar keine Verwechslung mit Ihrer eigenen Familie hätte vorkommen dürfen. Ich bin aber, und vielleicht ist es gut so, gar nicht zu einer solchen Erzählung gekommen, denn Frankreich, wo ich damals lebte, wurde von den Deutschen besetzt. Ich mußte mit meinen Kindern nach dem Süden, dabei habe ich eine Menge Manuskripte verloren oder verbrannt." 26

Geht es hier nur um Diskretion? War der neue Name so "beliebig"? Beruht die vergebliche Suche nach Stein und Straße auf einer Erinnerungslücke? Ich glaube nicht: Es geht zumindest auch um Fiktionalisierung, um narrative Ablösung vom Faktischen und um seine poetische

\footnotetext{
${ }^{23}$ Vgl. Christoph Bathelt: Ein Gedächtnisloch in der Mainzer Stadtgeschichte oder Ein Gedenkstein für Meta Cahn?! In: Gymnasium Moguntium. Blätter des Freundes- und Förderkreises des Rhabanus Maurus-Gymnasiums Mainz 56 (1993), S. 91-96. Dort auch das Faksimile des Briefes von Margarete Oppenheimer. Für präzisierende Informationen danke ich Barbara Prinsen-Eggert.

${ }^{24}$ Eine Studie über diesen möglicherweise zu Unrecht vergessenen Dichtersmann behalte ich mir im Rahmen meiner rheinhessischen Lokalforschungen vor; immerhin verdanken wir dem Büchner-Preisträger von 1923 Erzählwerke mit so ausdrucksstarken Titeln wie "Michael Hely, der Dorfteufel", "Am Stammtisch 'Zum faulen Hobel'" und "O domina mea".

${ }^{25}$ Besuche von Anna Seghers sind für u.a. 1947, 1962 und 1965 (allerdings erst nach der Publikation von "Zwei Denkmäler") nachgewiesen.

${ }^{26}$ Zitiert nach Glauert: Über ein vergessenes Denkmal in Mainz, S. 3.
} 
Aufladung. Die Verschleierung der historischen Fakten dient einer erzählerischen Wirkungsabsicht, die noch genauer betrachtet werden muß. Zunächst aber stellt sich die Frage, wie, wo und wann Anna Seghers einen 1940 in Frankreich verlorenen Text veröffentlichen konnte? Das führt uns zur Entstehungs- und Publikationsgeschichte von Zwei Denkmäler.

\section{III. "Mariage Blanc"}

Klaus Wagenbach veröffentlichte in seinem Verlag 1965 - unter dezidiert "gesamtdeutscher" Programmatik - eine gediegen ausgestattete und bis heute lesenswerte Anthologie mit dem Titel Atlas. Zusammengestellt von deutschen Autoren. ${ }^{27}$ Hier brachte er deutschsprachige Autoren mehrerer Generationen aus Bundesrepublik und DDR, aus Österreich, der Schweiz und aus dem Nachkriegsexil zusammen, die jeweils "ihren Ort" beschrieben. Von den berühmt gewordenen Texten sei vor allem Meine Ortschaft von Peter Weiss genannt ${ }^{28}$; aber auch Carl Zuckmayers schönes Erinnerungsbild Mainz, versunkene Stadt, das chronologisch genau dort endet, wo der nachfolgende Text, eben Zwei Denkmäler, beginnt: vor 1914. Die Skizze von Seghers hat dann in Wagenbachs Lesebuch. Literatur der sechziger Jahre (1968) zumindest in der Bundesrepublik zigtausendfache Verbreitung gefunden und ist ihrerseits eine Art Lesebuchklassiker, nicht nur in Deutschland, geworden - während sie, wie gesagt, in der DDR so gut wie unbekannt blieb. - Auf meine Anfrage hin hat Dr. Klaus Wagenbach vor wenigen Jahren noch ausdrücklich bestätigt, daß sein Atlas tatsächlich den Erstdruck von Zwei Denkmäler enthalte: "Ja! ich hatte Anna Seghers gebeten, einen Text für mich zu schreiben." Er sei "etwa Febr./März 65 geschrieben". Und es gebe "leider" keine "Korrespondenz" mehr darüber: "Zu oft umgezogen!"29 Diese Datierung ist ja auch textimmanent, durch die erinnernde Retrospektive aus der Zeit nach dem Zweiten Weltkrieg (und ihre Bedeutung für die Textkonstitution) völlig plausibel. Und doch: Es gibt einen Text gleichen Titels, der ganze zwanzig Jahre zuvor schon gedruckt wurde, und zwar in der Demokratischen Post in Mexico, am 1. August 1945. Ich verdanke seine Kenntnis Sigrid Thielking. ${ }^{30}$

\footnotetext{
${ }^{27}$ Später auch als Taschenbuchausgaben beim Deutschen Taschenbuch Verlag und in Wagenbachs Taschenbücherei.

${ }^{28}$ Mit Anna Seghers waren Peter Weiss und seine Frau Gunilla Palmstierna-Weiss 1964 bei einem Besuch in Buchenwald zusammengetroffen. Vgl. Jochen Vogt: Peter Weiss, Reinbek 1987, S. 90.

${ }^{29}$ Undatiertes Schreiben an den Verf. vom Juli 1993.

${ }^{30}$ Vgl. Zwei Denkmaeler. Aus einer unveroeffentlichten Novelle "Mariage Blanc". Von Anna Seghers. [Mit einer Zeichnung der Mainzer Altstadt und des Doms von Xavier Guerrero]. In: Demokratische Post. Organo de los Alemanes Demócratos de México y Centro-América, 1. August 1945, S. 8.
} 
Es handelt sich um eine sechzehnseitige Sondernummer: Zwei Jahre "Demokratische Post", die mit dem eigenen Jubiläum auch noch einmal die Niederwerfung des Hitler-Regimes und die Perspektiven für ein "neues" Deutschland feiert. Offensichtlich ist das Bemühen um eine würdigrepräsentative Ausgestaltung der Ausgabe, in der tonangebende Stimmen des antifaschistischen Exils zu Wort kommen. Bemerkenswert sind in diesem Sinne etwa der Leitartikel Rueckblick und Ausblick von Paul Merker (S.1); ein Vorabdruck aus Heinrich Manns Ein Zeitalter wird besichtigt (S.9), ein Bericht über die Bildung eines "demokratischen" Parteienblocks (KPD, SPD, CDU, LDP) in Berlin ${ }^{31}$ unter dem Titel Einigkeit macht stark von Alexander Abusch, oder etwa auch ein Gedenkblatt von Egon Erwin Kisch mit dem für unseren Zusammenhang bemerkenswerten Titel Denkmal fuer Teoberto Maler (S.7). ${ }^{32}$

Die Vermutung liegt nahe, daß die Redaktion auch die dem Blatt und seinem politischen Milieu eng verbundene Anna Seghers um einen Originalbeitrag bat. Aber was war noch in deren Schublade? - Der kurze Text Zwei Denkmaeler jedenfalls ist rechts unten auf Seite 8 plaziert, deren überwiegender Raum von einem bilanzierenden Aufsatz Probleme der zeitgenoessischen deutschen Literatur von Dr. Paul Mayer - bis 1936 Cheflektor bei Rowohlt, nun Lektor des Exilverlages el libro libre - eingenommen wird; hier findet sich ein emphatisches Lob von Seghers' Das Siebte Kreuz. ${ }^{33}$

\footnotetext{
${ }^{31}$ Vgl. Hermann Weber: Geschichte der DDR, München 1985, S. 81f.

${ }^{32}$ Den Festschrift-Charakter dieser Ausgabe unterstreichen auch zahlreiche Glückwunschadressen; besonders hervorgehoben sind diejenige Lion Feuchtwangers und die - offensichtlich von der Redaktion erbetene - Gratulation Thomas Manns, in der es heißt: "Ich hoffe sehr, dass meine Wuensche zu dem bevorstehenden Fest Ihrer Zeitschrift sie noch rechtzeitig erreichen. Ich habe die 'Demokratische Post' immer regelmaessig erhalten und stets mit Interesse durchgesehen. Es ist mir wohl keine Nummer vorgekommen, worin nicht irgend ein interessanter und anregender Artikel stand. Ich glaube, dass Ihre Zeitschrift in den beiden Jahren ihres Bestehens vielen Lesern, die mit uns in ihren tiefen Abscheu gegen den Faschismus und besonders seine deutsche Form, den National-Sozialismus, einig sind, ein Trost und eine Staerkung gewesen ist. - Besonders dankbar bin ich Ihnen fuer Ihr freundliches Gedenken zu meinem siebzigsten Geburtstag [...]." (S. 3) - In Form von Inseraten werden weitere Glückwüsche übermittelt, u.a. auch "Herzliche Gruesse" von "Dr. Laszlo Radvanyi, MEXICO, D.F." (S. 4). Ein anderes Inserat drückt den Wunsch aus, daß die vierzehntäglich erscheinende "Demokratische Post" nun "bald ein WOCHENBLATT wird" - hier unterzeichnet neben Hilde und Alexander Abusch, Walter Janka, Ludwig Renn und anderen auch "Anna Seghers" (S. 16). Deren Roman "Das Siebte Kreuz" wird zugleich in einer ganzseitigen Anzeige des Verlags "El libro libre" als "restlos vergriffen" gemeldet (S. 11).

33 "Die ganze Fuelle deutschen Schicksals umfasst der Roman 'Das Siebte Kreuz' von Anna Seghers. Hier hat die Form den Stoff verzehrt. Dieses Buch steht mitten in der Zeit und ragt hoch ueber seine Zeit hinaus. Wer dieses Buch liest, ist nicht allein, er ist mitten in Deutschland, unter Gefaehrten, Lebenden oder Toten. Er riecht den Apfelgeruch in den Apfelkammern, er hoert die Kinder ihre ewigen Reime singen, er steht mit den Metallarbeitern in der Fabrik und er harrt mit ihnen auf den Tag der Erloesung. Vielleicht wird dieses Werk unseren Nachfahren so bedeutungsschwer erscheinen, wie uns der Roman "Simplizissimus" von Grimmelshausen, in dem das Deutschland des dreissigjaehrigen Krieges weiterlebt. Hier wie dort sehen wir unser Land als Chaos, das vielleicht doch einmal den Stern gebiert."
} 
Überraschenderweise besitzen wir Zwei Denkmäler also in zwei deutlich unterschiedenen Fassungen; betrachten wir die bislang unbekannte ${ }^{34}$ ein wenig näher. Sicherlich war die Episode vom Tod der Mutter als Beginn einer längeren Erzählung konzipiert. Und zwar aus der Perspektive der Exilantin, die bei Kriegsende vermutet oder weiß, daß ihre Heimatstadt "vielleicht bis auf den Grund zerstoert worden ist". (Tatsächlich war die Innenstadt von Mainz zu $80 \%$ zerbombt. $^{35}$ ) Die Position des erzählenden Ichs ist - im Vergleich mit der Fassung von 1965 - nur schwach profiliert, die Zeitschichtung als Rahmen der Kernepisode fehlt fast völlig. Syntax und Sprachstil sind außerordentlich schlicht. Vorhanden ist hingegen das fiktionalisierende Motiv der Mutter, die "für ihre Kinder" (!) Milch holen will, hier ist es die "Frau des jüdischen Weinhändlers Gebhardt". Von einer Tochter oder deren Zukunft ist hingegen nicht die Rede; nur der Untertitel Aus einer unveröffentlichten Novelle "Mariage Blanc" läßt eine weiterreichende Intention der Autorin ahnen.

Tatsächlich hat Seghers schon 1940 an dieser Erzählung gearbeitet. Dem Verleger Wieland Herzfelde schreibt sie am 25. Januar aus Paris: "Meine Situation ist jetzt so, daß man mir tatsächlich und für einige Zeit helfen muß. Ich finde hier keinen Ausweg mehr, und ich habe große Lust, zu Euch zu kommen. [...] Aber, lieber Wieland, [...], [ich] bewahre [...] meine Ruhe und arbeite, ich will ein kleines Buch anfangen, das 'Mariage blanc' heißt, das bedeutet 'Paßheirat' (eine Heirat nur auf dem Papier). Es ist eine Liebesgeschichte, sehr traurig und fröhlich zugleich, ich glaube, Du hättest viel Vergnügen daran. Ich kann nicht erzähen, was ich zu schreiben begonnen habe. Ich glaube, das wäre etwas für den Film." ${ }^{36}$

Warum Mariage Blanc nicht ausgeführt wurde - oder ob das fertige Manuskript tatsächlich "verloren ging" ${ }^{37}$, und warum Seghers die Eingangspartie - der Hinweis auf die Zerstörung der Heimatstadt legt nahe, daß sie auf dem Kenntnistand von 1944/45 überarbeitet oder neu geschrieben wurde - im August 1945 in Mexico veröffentlichte, bleibt unklar. Zu diesem Zeitpunkt waren Das Siebte Kreuz (in englischer und deutscher Fassung) sowie die amerikanische Ausgabe von Transit schon veröffentlicht; Der Ausflug der toten Mädchen, 1943/44 abgeschlossen, wird 1946 im Aurora Verlag erscheinen.

\footnotetext{
${ }^{34}$ Sie ist wieder abgedruckt im Anhang von "Über Kunst und Wirklichkeit", Bd. IV: Ergänzungsband, Berlin 1979, S. $222 \mathrm{f}$.

${ }^{35}$ Vgl. Dieter Busch: Der Luftkrieg im Raum Mainz während des Zweiten Weltkriegs 1939-45, Mainz 1988, S. Vf.

${ }^{36}$ Anna Seghers/Wieland Herzfelde: Gewöhnliches und gefährliches Leben. Ein Briefwechsel aus der Zeit des Exils 1939-1946. Hrsg. im Auftrag der Akademie der Künste der DDR von Ursula Emmerich und Erika Pick, Darmstadt und Neuwied 1986, S. 40f.
} 
In diesem Zeit- und Werkkontext kann die enge Motiv-Verbindung der Zwei Denkmäler zum Siebten Kreuz (der Dom, der Rhein), zu Transit (die Scheinheirat, die "Schiffe und fernen Städte") und vor allem zum Ausflug der toten Mädchen (die Schulzeit, die Stadt mit ihren Straßen, der Bombentod einer Frau, ein überlebendes Kind usw.) nicht verwundern. Überraschend ist nur, daß diese Intertextualität der Zweitfassung von 1965 sehr viel stärker eingeschrieben ist als der ersten - ein Werk der Erinnerung, das insofern noch einen genaueren Blick verdient.

\section{Verwischte Spuren}

Die Komplexität der Zeitschichtung, das dichte Motiv- und Symbolgeflecht, die intensive Leserwirkung von Zwei Denkmäler verdanken sich zweifellos erst dieser reécriture aus der Kraft der Erinnerung und der objektiven Phantasie. Erst aus Sicht der Fünfundsechzigjährigen ist dieser Zoom durchs 20. Jahrhundert möglich. Und was sich zunächst als faktualer Bericht darstellte, erweist sich nun als kunstvoller Spiegeleffekt von facts und fiction.

Setzen wir nochmals bei der auffälligen Fiktionalisierung der Kernepisode an. "Hieß die Straße Bonifaziusstraße? Hieß sie Frauenlobstraße? Das weiß ich nicht mehr." Die Bonifaziusstraße ist die Verlängerung, die Frauenlobstraße eine Parallelstraße jener anderen, von Kindheit an vertrauten Straße, deren Name Anna Seghers hier eher ausspart als wirklich vergessen hat: eben die Schulstraße. Und auch die Ereignisse von 1918 in Mainz sind unvergessen: "Meine ersten Bombenangriffe habe ich am Ufer des Rheins erlebt", notierte Anna Seghers noch im Juni 1938 in Paris. ${ }^{38}$ Die Erinnerungsschwäche der Autobiographin erweist sich als metonymische Ausweichtaktik, die aber zugleich eine semantische und symbolische Aufladung des Erzählten möglich macht. Winfried Bonifatius, irischer Missionar der Deutschen, von 746 bis 754 Erzbischof von Mainz, ist eng mit dem ersten Denkmal, dem mächtigen Dom verbunden. Die Frauenlobstraße spricht semantisch-symbolisch für sich selbst (auch wenn sie historisch an den Minnesänger Heinrich von Meißen - genannnt Frauenlob - erinnert, der 1318 in Mainz verstarb) und kann offensichtlich mit dem zweiten Denkmal assoziiert werden. Die Opposition, die hier zu erkennen ist, wird andererseits wieder eingeebnet oder verschleiert. Eppstein - der Name also, der das allzu jüdische Cahn und den "beliebigen fremden Namen"

\footnotetext{
${ }^{37}$ Vgl. den Kommentar ebda., S. 187.

${ }^{38}$ Anna Seghers: Sechs Tage, sechs Jahre. Tagebuchseiten, in: Neue deutsche Literatur, H.9/1984, S. 9. - Für diesen Hinweis danke ich Alexander Stephan.
} 
Gebhardt ersetzt, - ist ein verbreiteter deutschjüdischer Familienname, aber auch eine rheinhessische Burg und Stadt. Fünfmal hielten zwischen 1060 und 1305 die Grafen von Eppstein den Posten des Mainzer Erzbischofs besetzt. Unter ihnen hatte Siegfried III. von Eppstein (12301249), dessen Grabmal sich heute noch im Dom befindet, als Erbauer des Westchores, den er 1239 weihte, eine besonders enge Beziehung zu diesem Baudenkmal. ${ }^{39}$

Die symbolische "Taufe" der Frau Cahn, die nun Eppstein heißt, belegt jedenfalls, daß Seghers keine fundamentale Opposition von Christentum und Judentum aufbaut; die jüdische Mutter bzw. das archetypisch ausgestaltete Lob der Mutter - wird vielmehr durch die Namengebung in den heimatlichen (und dominant christlichen) Traditionszusammenhang eingebunden. Das ließe sich, in Seghers' lebensgeschichtlicher Perspektive durchaus plausibel, als nostalgische Rückwendung auf eine deutsch-jüdische Symbiose interpretieren. Aber vielleicht muß man auch, grundsätzlicher, Anna Seghers' ganz "eigene Art" in Rechnung stellen, "Spuren zu verwischen und andere auszulegen" - auf die Sonja Hilzinger vor kurzem hingewiesen hat. ${ }^{40}$ Dieser Hinweis führt uns jedenfalls, in einer Art von interpretatorischem Zickzack, noch einmal auf die Textfassung von 1945 zurück.

V. "... missing from a camp near Lublin"

\footnotetext{
${ }^{39}$ Vgl. Fritz Arens: Der Dom zu Mainz, Darmstadt 1982, S. 90. - Siegfrieds III. Denkmal, genauer: die Grabplatte aus grauem Sandstein, die nach wie vor an einem Pfeiler nahe des Aufgangs zum Ostchor angebracht ist, figuriert im "Siebten Kreuz" offensichtlich in der Episode, die erzählt, wie der verfolgte Georg Heisler sich im abendlichen Dom einschließen läßt: "Er suchte sich ein Versteck. Er sprang von einem Pfeiler zum andern. Er duckte sich, als sei er noch immer beobachtet. An dem Pfeiler, vor dem er jetzt hockte, lehnte, gleichmütig aus seiner Grabplatte über ihn hinwegsehend, ein runder gesunder Mann, auf seinem vollen Gesicht das dreiste Lächeln der Macht. In jeder Hand eine Krone, unbemerkt von Georg, krönte er unablässig zwei Zwerge, die Gegenkönige des Interregnums. Georg sprang in einem Satz, als seien die Zwischenräume belauert, zu dem nächsten Pfeiler. Er sah an dem Mann hinauf, desen Kleider so reich waren, daß er sich hätte hineinwickeln können. Er fuhr zusammen. Ein menschliches Angesicht, das sich über ihn beugte voller Trauer und Besorgnis. Was willst du denn noch, mein Sohn, gib auf, du bist schon am Anfang zu Ende. Dein Herz klopft, deine kranke Hand klopft. [...] Er konnte noch immer von seinem Platz aus den Mann am Eckpfeiler erkennen. Trotz der Dunkelheit war das Gesicht von weitem eher noch klarer. Auf den gekrümmten Lippen das letzte, das äußerste Angebot: Friede statt Todesangst, Gnade statt Gerechtigkeit." (Anna Seghers: Das siebte Kreuz. Roman, Darmstadt/Neuwied 1973, S. 53f. - Hervorhebung J.V.) - Auf Siegfried III. von Eppstein deutet spezifisch die Erwähnung der beiden "Gegenkönige" Wilhelm von Holland und Heinrich Raspe von Thüringen. Analog ist das gegenüberstehende Grabmal des Erzbischofs Peter von Aspelt (1306-1320) geformt, der freilich gleich drei "Zwerge" krönt. (Vgl. Fritz Arens: Der Dom zu Mainz, Darmstadt 1982, S. 88-92; August Schuchert/Wilhelm Jung: Der Dom zu Mainz. Ein Handbuch, 3. Aufl. Mainz 1984, S. 26-29.) Ich gehe davon aus, daß Seghers die Versteckszene nicht im strengen Sinne empirisch-topographisch, sondern als freie Montage von Erinnerungsbildern (oder auch Bildvorlagen) konzipiert hat. - Sich ungesehen in den Dom einschließen zu lassen, an Werktagen kurz vor 18.30 Uhr, ist auch heute noch kein Problem; es gibt jedoch, wie ich inzwischen weiß, angenehmere Lokalitäten ganz in der Nähe.

40 Sonja Hilzinger: Anna-Seghers-Kontexte, in: Horizonte. Rheinland-pfälzisches Jahrbuch für Literatur 3, Frankfurt/M. 1996, S. 212.
} 
In den Briefen von Anna Seghers aus Mexiko macht sich um eben jene Zeit - ich zitiere aus Alexander Stephans Analyse der FBI-Akte Seghers - ein Thema geltend, "das die SeghersForschung bislang mit kaum einem Wort erwähnt hat: die Sorge um das Schicksal der Mutter, die im Frühjahr 1942, zweiundsechzigjährig, mit einem der großen Transporte von Juden aus Mainz in das Ghetto Piaski bei Lublin verschleppt worden war." ${ }^{41} \mathrm{Zu}$ Beginn des Jahres 1945 wird aus der Sorge eine schreckliche Gewißheit. "I do not have to tell you" - heißt es (vom FBI ins Englische gebracht) in einem Brief von Anna Seghers in die Schweiz vom 27. 3. - "how desperate I am about what happened to Mother. Just now Mother could live with us without any financial difficulty. I wrote [ausgeschwärzt] ... that we were able to cable Mother that we secured her a Mexican visa. She even had a Swiss visa but the Nazis would no longer let her get out. Gjury wrote me that you were sending her parcels, until the mail was returned to you; we do not have to delude ourselves as to what this means..." Und eine Woche zuvor: "Mother... has been declared missing from a camp near Lublin." ${ }^{42}$ Ähnlich bereits in einem Brief an Kurt Kersten vom 2. 1. 1945: "Wir haben verteufelte Nachrichten. Meine Mutter, von der ich schon Jahre nichts hörte, wurde zuletzt in ein KZ nach Polen transportiert, wo sie vermutlich zugrunde gegangen ist." ${ }^{43}$ Die Fakten dieser Deportation vom 20. März 1942, die in das polnische Ghetto Piaski (und von dort aus möglicherweise ins Vernichtungslager Sobibor oder Belzec) führte, sind - soweit möglich inzwischen ermittelt worden. ${ }^{44}$ Unter den eintausend hessischen Juden und Jüdinnen, davon allein 450 aus Mainz, befand sich neben der 62 Jahre alten Hedwig Reiling auch die Lehrerin Johanna Sichel. ${ }^{45}$ Ihr setzt die ehemalige Schülerin schon im Ausflug der toten Mädchen, 1943/44 niedergeschrieben, ein Denkmal - zugleich wird, thematisch weniger auffällig, aber durchaus explizit, auch schon die Deportation der Mutter erwähnt: "Sie stand vergnügt und aufrecht da, bestimmt zu arbeitsreichem Familienleben, mit den gewöhnlichen Freuden und Lasten des

\footnotetext{
${ }^{41}$ Alexander Stephan: Im Visier des FBI. Deutsche Exilschriftsteller in den Akten amerikanischer Geheimdienste, Stuttgart/Weimar 1995, S. 479.

${ }^{42}$ Ebda.

${ }^{43}$ Zitiert nach: Anna Seghers. Eine Biographie in Bildern, S. 143.

${ }^{44}$ Frank Wagner: Deportation nach Piaski. Letzte Stationen der Passion von Hedwig Reiling, in: Argonautenschiff 3 (1994), S. 117-126. Vgl. auch: Bruno Lowitsch/Roland Siegrist: "Fertig zur Abwanderung". Der letzte Weg der Hedwig Reiling in Mainz am 20. März 1942, in: Mainz 10 (1990), H. 1, S. $120 \mathrm{ff}$.

${ }^{45}$ Vgl. die Deportationslisten mit beiden Namen, in: Anna Seghers. Eine Biographie in Bildern, S. $31 \mathrm{f}$.
} 
Alltags, nicht zu einem qualvollen, grausamen Ende in einem abgelegenen Dorf, wohin sie von Hitler verbannt worden war."46

Bald danach - und lange vor dem Druck der Erzählung 1946 - bemüht sich Seghers, den Ausflug der toten Mädchen als Filmversion neu zu konzipieren und zwar in einer neuartigen Retrospektive; in einem Brief nach Santa Monica vom 23. 9. 44 schlägt sie vor, "to build it up as a post-war play. [...] I return home, the city is completely changed, here traces still of the bombardment, there disfiguring reconstruction. Where my school was, there is something else entirely. I looked for the girls of my class. They are not to be found. [..] Here in the film or dramatic version in any case a girl must have been saved, perhaps one who was at that time very much endangered, very broken, and perhaps in this way or that way through a completed action which must be connected with the beginning of the story, must come through to us and show her new situation." ${ }^{47}$

Die Demokratische Post, als deren regelmäßige Leserin wir Anna Seghers ansehen dürfen, brachte seit Frühjahr 1945 eine Artikelserie über die langsam bekannt werdenden Greuel der Konzentrationslager: Ein deutscher General über Maidanek (15. April), Die Bestien von Kiew (2. Mai), Augenzeugenberichte aus den Nazi-Höllen (17. Mai), Die Massenmorde von Ossiecim (15. Juli). Die Skizze Zwei Denkmäler wird am 1. August 1945 in rudimentärer Form publiziert (weil die Redaktion etwas brauchte? weil die Mutter-Tochter-Thematik zu schmerzhaft war, um sie weiter zu verfolgen?); möglicherweise wird das Projekt Mariage Blanc erst jetzt aufgegeben. Die fröhliche Liebesgeschichte mag nun zu frivol erscheinen; einzelne Motive sind überdies schon im Ausflug verbraucht; könnten jedoch bei Bedarf in der geplanten Filmfassung wieder aufgenommen werden. Das ist aber, wie wir wissen, nicht geschehen. Es scheint so, als habe erst Klaus Wagenbachs Anfrage von 1965 den Impuls gegeben, das alte, halb vergessene Motiv des mütterlichen Opfers - hier im Doppelsinn von sacrifice und victim - und die Frage nach der Zukunft der Tochter wieder aufzugreifen.

VI. Geschichte(n) eines Adjektivs

\footnotetext{
${ }^{46}$ Anna Seghers: Der Ausflug der toten Mädchen, in: A.S.: Der Bienenstock. Ausgewählte Erzählungen in zwei Bänden, Berlin/DDR 1953, Bd. 1, S. 180. - Vgl. hierzu Sonja Hilzinger: Anna Seghers: "Der Ausflug der toten Mädchen", in: Erzählungen des 20. Jahrhunderts, Bd. 2, Stuttgart 1996, S. $38 f$.

${ }^{47}$ Zitiert nach Stephan: Im Visier des FBI, S. 480.
} 
Das Schicksal des Kindes, das erst im letzten Satz ein Mädchen genannt wird, also einer jungen jüdischen Frau, die dem Terror des Nationalsozialismus ausgesetzt sein wird, ist nun eingebunden in die historische Retrospektive, die zuerst in Seghers' Filmprojekt auftauchte, also in einen lebens-, schaffens- und zeitgeschichtlichen Rückblick der Autorin, in dem dann auch die thematischen Querverbindungen zu Transit, zum Siebten Kreuz und zum Ausflug der toten Mädchen hervortreten (dürfen). Die Mutter-Tochter-Konstellation in Zwei Denkmäler aber spiegelt, wie indirekt und fiktional kamoufliert auch immer, doch auch die Beziehung von Hedwig und Netty Reiling. Explizit macht der Text das nur in einem einzigen Adjektiv: Es geht, in mehrfach gebrochener Perspektive, eben auch um Frau und Tochter des jüdischen Kunsthändlers Reiling. Die ungewisse Zukunft des jüdischen Mädchens mit Namen Eppstein enthält - unausgeführt - die beiden möglichen Schicksalswege der Mutter wie der Tochter Reiling: Deportation und Ermordung oder Flucht und Überleben im Exil. ${ }^{48}$ Diese Spiegelung ist deutlich und subtil zugleich; vielleicht deswegen, weil sie - wie der berühmte "entwendete Brief" bei E. A. Poe - an der (un)auffälligsten, weil (un)wahrscheinlichsten Stelle verborgen ist: verschlüsselte Autobiographie innerhalb der offenen.

Erklären kann man dies Verfahren gewiß aus Seghers' vielfach bezeugter Abwehr gegen biographische Nachfragen, aus einer Art von Biographie-Verweigerung, wenn man so sagen darf $^{49}$, genauer vielleicht aus ihrer nur selten durchbrochenen Weigerung, die eigene jüdische Identität zu thematisieren - eine persönliche Disposition, die durch jahrzehntelange Erfahrungen in der Kommunistischen Partei verfestigt und nach der Rückkehr durch den systembedingt latenten Antisemitismus in der DDR verstärkt worden sein dürfte. ${ }^{50}$

\footnotetext{
${ }^{48}$ Es ist fast überflüssig, darauf zu verweisen, daß die gruppenbildähnliche Kombination verschiedener Lebenswege, Schicksale und Haltungen und die Schürzung von biographischen "Knotenpunkten" (Helmut Scheuer) ein bevorzugtes Kompositionsprinzip von Anna Seghers ist. Vgl. in diesem Sinne auch den sehr narrativ geprägten Aufsatz "Frauen und Kinder in der Emigration", in: Anna Seghers/Wieland Herzfelde: Gewöhnliches und gefährliches Leben, S. 128-145.

49 Zum gesamten Problemkomplex die differenzierten Überlegungen von Helmut Scheuer: Biographik und Literaturwissenschaft: Konstruktion und Dekonstruktion. Anna Seghers und ihre Biographen, in: Argonautenschiff 4 (1995), S. 245ff.

${ }^{50}$ Zum Problem vgl. Hinweise bei Hans Albert Walter: Anna Seghers. In: Hans Jürgen Schultz (Hrsg.): Es ist ein Weinen in der Welt. Hommage für deutsche Juden unseres Jahrhunderts, Stuttgart 1990, S. 407ff., sowie Sonja Hilzinger: Anna-Seghers-Kontexte, in: Horizonte. Rheinland-pfälzisches Jahrbuch für Literatur 3, Frankfurt/M. 1996, S. $200 \mathrm{f}$
} 
Außerordentlich wirkungsvoll, in seiner Einfachheit fast genial ist nun aber die "offene" Form des Textes ${ }^{51}$, Seghers' Kunstgriff, das Schicksal der fiktionalisierten Figur, die sie ins Zentrum stellt, eben nicht zu erzählen, sondern auszusparen und die Ausfüllung dieser Ellipse, die Lösung des Knotens den Leserinnen und Lesern zu überlassen. Die 1940 einmal geplante Geschichte, die zweifellos eine Rettungsgeschichte gewesen wäre ${ }^{52}$, läßt sich allein aus Gründen historischen Wissens nicht mehr so erzählen. Der mögliche Tod des Mädchens Eppstein wie der faktische Tod von Hedwig Reiling im Ghetto oder Lager, also die komplementäre Vernichtungsgeschichte, kann hingegen aus anderen Gründen nicht erzählt werden. Damit reagiert Seghers - ich möchte behaupten: eher instinktiv - auf die grundsätzliche Aporie aller Holocaust-Literatur: der innerste Kern des Terrors, die letzten Leidenserfahrung entziehen sich dem Erzählen schlechthin, oder jedenfalls der chronikalisch-lebensgeschichtlichen Erzählweise, die Anna Seghers stets gepflegt und entwickelt hat. Und doch: "Weil er ein Jude war, starb mein Vater in Auschwitz", schrieb 1987 die französische Philosophin Sarah Kofman. "Wie könnte ich nicht darüber sprechen? Und wie kann ich darüber sprechen?"53 $\mathrm{Ob}$ und wie Leser und Leserinnen die von Seghers verschwiegene, aus- und aufgesparte Geschichte erzählen, ob und wie sie die Lücke im Text, hinter der "die unermeßliche Leerstelle" Auschwitz steht ${ }^{54}$, ihrerseits auffüllen, das wird entscheidend davon abhängen, wie sie jenes Wort jüdisch lesen. "Schriebe ich meine Autobiographie," vermerkt Isaak Babel einmal, "so würde ich sie 'Geschichte eines Adjektivs' nennen." ${ }^{55}$

\section{Die Kraft der Schwäche}

\footnotetext{
51 "Offenheit" ist im Jahr des berüchtigten 11. Plenums des ZK der SED sowohl formal wie politisch ein Reizwort. Zu Seghers' literaturpolitischer Position vgl. Zehl Romero: Anna Seghers, S. 121f.

${ }^{52}$ Vgl. hierzu die Paraphrase - oder besser gesagt: 'Fortschreibung' durch Christa Wolf, von der man wohl vermuten darf, daß sie auf Gesprächen mit Anna Seghers selbst basiert: "Nach diesem Rezept [Balzacs] fängt sie [...] eine Geschichte an zu schreiben, - wer wird sie je drucken, wer in deutscher Sprache lesen können und wollen -, die sie 'Weiße Hochzeit' nennt: eine jener vorgetäuschten Hochzeiten, die, nicht so selten, zum Schein geschlossen wurden; zum Beispiel kann die Braut bestimmte Papiere brauchen, um zu ihrem richtigen Bräutigam zu gelangen, Papiere, die sie allerdings nur als verheiratete Frau bekommt. Die geplante wirkliche Hochzeit aber wird niemals begangen, dagegen erweist sich die falsche als ernst und dauerhaft. - Das Manuskript der 'Weißen Hochzeit' ist verlorengegangen. Wäre es erhalten, hätten wir zweifellos ein Zeugnis mehr für die Eigenart der Segherschen Fabeln: merkwürdige Zufälle brechen in die Lebensläufe ihrer Personen ein und geben ihnen eine andere Richtung; was die Menschen erstreben, zieht sich wie unter einem Zauber von ihnen zurück, aber was sie dafür erhalten, ist nicht schlechter, auf jeden Fall ist es ihr wirkliches Leben." (Christa Wolf: Glauben an Irdisches, 1969, in: Lesen und Schreiben. Neue Sammlung. Essays, Aufsätze, Reden, Darmstadt /Neuwied 1980, S. 128.)

${ }^{53}$ Sarah Kofman: Erstickte Worte, Wien 1988, S. 27.

${ }^{54}$ Maurice Blanchot: L'Ecriture du désastre, zitiert nach Kofman: Erstickte Worte, S. 11.
} 
In dieser Perspektive könnte Anna Seghers' autobiographische Miniatur eine neue Aktualität gewinnen, genauer gesagt: innerhalb der lebhaften Diskussion über die Formen und Gesetzmäßigkeiten des "kulturellen Gedächtnisses" im allgemeinen ${ }^{56}$, besonders jedoch über die Formen und ästhetisch-ideologischen Funktionen von "Denkmälern" und die mit ihnen betriebene "Erinnerungspolitik". ${ }^{57}$ Derartige Debatten werden derzeit sowohl - international und interdisziplinär - in den Kulturwissenschaften geführt wie auch in der politisch-publizistischen Öffentlichkeit ausgetragen - hier zumeist in Form teils sachlicher, teils polemischer Kontroversen um symbolträchtige Gedenkstätten. (Dies geschieht nicht nur, aber doch auffallend oft in der Metropole der "Berliner Republik", was deren Bedürfnis nach - und die Schwierigkeiten mit einer neuen und konsensfähigen offiziellen "Erinnerungspolitik" anzeigt.)

Wenn Denkmäler im konkreten Sinn ein privilegierter Gegenstand solcher Überlegungen sind, so ist der Holocaust zweifellos der negativ transzendentale Horizont aller einschlägigen Überlegungen. ${ }^{58}$ "Nach" und gegenüber "Auschwitz" potenzieren sich die grundsätzlichen Fragen, die an jedes Denkmal gestellt werden können, fast aporetisch: Wie ist das kulturelle Gedächtnis beschaffen, dem dies oder jenes Denkmal seine Existenz verdankt? Welche Geschehnisse hat es bewahrt, welche nicht? Aus der Perspektive der Täter, der Zeugen oder der Opfer? Wie läßt sich - läßt sich überhaupt - geschehenes Unrecht, erlittenes Leid mit ästhetischen Mitteln zum Ausdruck bringen? Verdecken Symbolisierungen und Abstraktionen wesentliche Differenzen (z.B. zwischen verschiedenen Opfern)? Dienen sie gar einer Entlastung der Täter? Wie ist zu erreichen, daß die Monumente nicht im schlechten Sinne "monumental" bleiben, sondern wirksame Impulse für aktive Erinnerungsarbeit geben?

Natürlich will ich nicht behaupten, daß Seghers' kleiner Text solch grundsätzliche Fragen beantworten kann. Aber es könnte ergiebig sein, ihn vor der Folie dieser Fragestellungen zu lesen, die er auf seine Weise - poetisch und implizit - vorwegnimmt. Zunächst einmal sind die zwei Denkmäler in Zwei Denkmäler scheinbar entgegengesetzte Monumente kirchlicher und staatlicher Macht einerseits, persönlicher Ohnmacht andererseits. (Da der Dom aber nicht nur

\footnotetext{
${ }^{55}$ Vgl. Elke Schmitter: Heimwege in die Fremde. "Geschichte eines Adjektivs" - jüdische Autobiographien am Ende des 20. Jahrhunderts, in: Die Zeit, 8. 11. 1996, Literatur, S. 1.

${ }^{56}$ Vgl. Aleida Assmann/Dietrich Harth (Hrsg.): Mnemosyne. Formen und Funktionen der kulturellen Erinnerung, Frankfurt/M. 1991; dies. (Hrsg.): Kultur als Lebenswelt und Monument, Frankfurt/M. 1991.

57 Reinhart Koselleck/Michael Jeismann (Hrsg.): Der politische Totenkult. Kriegerdenkmäler in der Moderne, München 1994; Peter Reichel: Politik mit der Erinnerung. Gedächtnisorte im Streit um die nationalsozialistische Vegangenheit, München/Wien 1995.
} 
Monument, also "Erinnerungszeichen" ist, sondern auch als Versteck oder Schutzraum dienen kann, wie wir mindestens aus dem Siebten Kreuz wissen, wird der Gegensatz ein Stück weit aufgelöst.) Der "kleine flache Gedenkstein" markiert als "Erinnerungszeichen" zunächst einmal einen "Tatort" als "Gedächtnisort" ${ }^{15}$ und gilt dem Andenken eines individuellen Opfers. Dies Gedenken aber ist von Vergessen bedroht, so wie - jedenfalls in der Version des Textes - der Gedenkstein nicht mehr auffindbar ist: Von den Nazis zerstört? Unter Trümmern begraben? Im Nachkriegs-Wirtschaftswunder zubetoniert? "Auf ein Gedächtnis der Orte aber ist", wie Aleida Assmann in einem ähnlichen Zusammenhang vemerkt, "ohne flankierende Maßnahmen wenig Verlaß. [...] das sprichwörtliche Gras des Vergessens tut seine Wirkung. Im Gegenteil bedarf es ungeahnter Anstrengungen, die Lücke, die Leerstelle als Spur der Vernichtung zu bewahren."60 Es geht, anders gesagt, darum, das räumliche Erinnerungszeichen in "die Zeitdimension" zurückzuführen, in der allein "Erinnerung lebendig erhalten" werden kann. ${ }^{61}$ Das scheint in diesem Fall, wo die ästhetische Gestalt des Denkmals selbst eine Leerstelle bildet (im Text ist es nicht mehr auffindbar, in der Realität ohnehin unbeschriftet), besonders notwendig, aber vielleicht auch besonders produktiv - "gerade diese Leere schafft Raum für eigene Imaginationen". $^{62}$ (Die strukturelle Analogie zu neueren "Gegen-Denkmälern", etwa von Jochen und Esther Gerz u.a., die sich selbst unlesbar machen oder ihr eigenes Verschwinden inszenieren, ist augenfällig. ${ }^{63}$ )

Welches Medium aber wäre für jene Anstrengung, für die neu zu stiftende, paradoxe Überlieferung geeigneter als die "Stimme der Erzählerin" (Benjamin), zu der eben auch das Verstummen gehört? Sie koppelt das Gedenken ab vom ursprünglichen ideologischen Diskurs, in dem die Opfer posthum militarisiert und zugleich als Zeugen vorgeblich deutschen Friedenswillens instrumentalisiert wurden; sie legt nahe, das Denkmal der Mutter metonymisch als eines für die Tochter zu lesen, deren Schickal in aller Ungewißheit - im Exil oder in "Auschwitz" - wiederum stellvertretend für die Schicksale Unzähliger stehen kann.

\footnotetext{
${ }^{58}$ Vgl. zuletzt Nicolas Berg, Jess Jochimsen, Bernd Stiegler (Hrsg.): Shoah. Formen der Erinnerung. Geschichte, Philosophie, Literatur, Kunst, München 1996.

${ }^{59}$ Zur Begrifflichkeit vgl. Aleida Assmann: Das Gedächtnis der Orte, in DVjs., Sonderheft 1994, S. 17-35.

${ }^{60}$ Ebda., S. 32f.

${ }^{61}$ Vgl. Andreas Huyssen: Faszination des Monumentalen: Geschichte als Denkmal und Gesamtkunstwerk, in: Hartmut Böhme/Klaus R. Scherpe (Hrsg.): Literatur und Kulturwissenschaften. Positionen, Theorien, Modelle, Reinbek 1996, S. 283f.

${ }^{62}$ Reichel: Politik mit der Erinnerung, S. 140.

${ }^{63}$ Vgl. den Abschnitt über "Unsichtbare und ephemere Denkmäler: Erinnerung an das Vergessen", ebda., S. 119ff.
} 
In der narrativen Umformung ist ein anti-"monumentales" Erinnerungszeichen, ein "GegenDenkmal" entstanden, "das keinen Sinn mehr stiftet, wie bislang üblich, sondern das die Frage nach dem Sinn selber in Frage stellt. [...] Es ist der Zwang zur Reflexivität - oder zur 'Besinnung' - der von solchen Denkmälern ausgeübt wird. Er verweigert Sinnstiftung und Sinnfindung zugleich." 64

Die von der Autorin Anna Seghers einstmals ersonnene und begonnene Geschichte der Zwei Denkmäler, die erzählen sollte, "was aus dem Mädchen geworden ist" (aller Vermutung nach eine Rettungsgeschichte), ist - gewissermaßen als drittes Denkmal - selbst der Gewalt der Geschichte zum Opfer gefallen. Den kleinen Erinnerungstext, der uns hier beschäftigt hat, könnte man als ein viertes Denkmal begreifen, das Leserinnen und Leser auf seine zurückhaltende Weise zur "Besinnung" auffordert.

In diesem Sinne spricht Zwei Denkmäler ganz zuletzt, ziemlich zurückhaltend und (wie die Literaturwissenschaftler sagen) selbstreferentiell auch von der Kraft der (schwachen) Literatur, vom dichterischen Selbstbewußtsein noch in dunkelsten Zeiten. Exegi monumentum aere perennius: "Aufgerichtet habe ich ein Denkmal, dauerhafter als Erz" - das Selbstbewußtsein dichterischer Monumentalität, das aus den zweitausendjährigen Versen des $\operatorname{Horaz}^{65}$ spricht, scheint freilich für unseren Zusammenhang ebenso unpassend wie Hölderlins "Was bleibet aber, stiften die Dichter", das Martin Heidegger so gern zitierte. ${ }^{66}$ Vielleicht aber darf zum Schluß doch eine Überlegung angeführt werden, die der Turiner Philosoph Gianni Vattimo urprünglich unter Bezug auf Heideggers Kunstauffassung angestellt hat. Eine "monumentale Sicht des Kunstwerks" vollzieht sich im Gegenwartshorizont allein "in der Weise des Peripheren". "Es geht aber um ein Bleiben, das eher durch den Grundzug des Überrestes als jenen des aere perennius gekennzeichnet ist. Das Denkmal ist zwar zum Überdauern geschaffen, aber nicht als volle Anwesenheit dessen, wovon es die Erinnerung trägt; es bleibt vielmehr eben als Erinnerung [...]. Die Techniken der Künste zum Beispiel [...] können als [...] Kunstgriffe betrachtet werden, welche das Werk in einen Überrest verwandeln, in ein Denkmal, zur Dauer fähig, weil von

\footnotetext{
${ }^{64}$ Reinhart Koselleck: Einleitung, in: Koselleck/Jeismann (Hrsg.): Der politische Totenkult, S. 18f.

${ }^{65}$ Quintus Horatius Flaccus: Carmina III, 30

${ }^{66}$ Vgl. etwa Martin Heidegger: Hölderlin und das Wesen der Dichtung, in: M.H.: Erläuterungen zu Hölderlins Dichtung, 5. Aufl. Frankfurt/Main 1981.
} 
Anfang an in die Form dessen, was tot ist, gegossen; fähig nicht aufgrund seiner Stärke, sondern seiner Schwäche." ${ }^{67}$

\section{$\underline{\text { Über den Autor }}$}

Prof. Dr. Jochen Vogt, geb. 1943, Autor der "Einladung zur Literaturwissenschaft", ist Professor für Germanistik/Literaturwissenschaft und Literaturdidaktik an der Universität Essen. Seine Hauptarbeitsgebiete umfassen die deutsche und europäische Literatur des 20. Jahrhunderts, Literaturtheorie (insbesondere Narratologie) und Literaturdidaktik.

e-mail: jochen.vogt@uni-essen.de

\section{Wichtigste Publikationen:}

- Struktur und Kontinuum. Hans Henny Jahnns "Fluß ohne Ufer",1970

- Korrekturen - Versuche zum Literaturunterricht, 1974

- Einführung in die deutsche Literatur des 20. Jhs.(Hg.), 3 Bde, 1977ff.

- Thomas Mann. "Buddenbrooks", 1982

- Heinrich Böll, 1987

- Peter Weiss, 1987

- Aspekte erzählender Prosa. Erzähltechnik und Romantheorie. Neufassung 1990

- "Erinnerung ist unsre Aufgabe". Literatur-Moral-Politik nach 1945, 1991

- Der Kriminalroman. Poetik-Theorie-Geschichte (Hg.), 1998

\footnotetext{
${ }^{67}$ Gianni Vattimo: Ornament (als) Denkmal, in: G.V.: Das Ende der Moderne, Stuttgart 1990, S. 94.
} 